\title{
VALOR PRONÓSTICO DE LA AFECTACIÓN MICROSCÓPICA DE LA VENA RENAL EN EL CÁNCER DE CÉLULAS RENALES
}

\author{
Gloria Bocardo Fajardo, Ramón Arellano Gañán, Lucía González López', Inmaculada \\ Fernández González, Julia Blanco González², Pablo Garrido Abad, Almudena Coloma del \\ Peso e lgnacio Pereira Sanz.
}

Servicio de Urología. Hospital de la Princesa. Madrid. Servicio de Anatomía Patológical. Hospital de Alarcos. Ciudad Real. Servicio de Anatomía Patológica². Hospital Clínico San Carlos. Madrid. España.

\begin{abstract}
Resumen.- OBJETIVO: En el carcinoma de células renales, la TNM define el estadio T3b como afectación macroscópica de la vena renal o cava infradiafragmática. El valor pronóstico de la invasión microscópica de la pared de la vena no se contempla. Analizamos la repercusión de ésta en la evolución de estos tumores, en estadios localizados y localmente avanzados.
\end{abstract}

MÉTODOS: De 257 carcinomas de células renales operados (Enero de 1989 / Julio de 2002), se analizan retrospectivamente 241 lexcluímos los estadios IV y los casos con enfermedad de Von-Hippel-Lindau). El

\section{CORRESPONDENCIA}

Gloria Bocardo Fajardo

Eugenia de Montiio, 55. Portal I. 1으 C 28025 Madrid. (España).

bocardo@telefonica.net

Trabajo recibido: 19 de junio 2008 seguimiento mediano es de 50,96 meses. Tenemos datos de la histología de la vena en 216 casos. Comparamos el grupo con invasión microscópica de la pared (22 casos) frente al resto (n: 194).

RESULTADO: En el grupo con afectación de la pared, la edad media es 65,02 años. El tamaño medio es 9 $\mathrm{cm}$ (significativamente superior, $p<0,001$ ). El 72,7\% presentan trombo frente al 6,2\% del segundo grupo. El $77,3 \%$ corresponden a tumores de células claras. El $42,9 \%$ tienen un grado 2 de Fuhrman. El riesgo de desarrollar metástasis (HR: 4,86$)$ y de éxitus (HR: 6,49$)$ es significativamente superior en el grupo con afectación de la pared. Este riesgo es superior ante la presencia de trombo (HR: 7,22 y HR: 8,38, respectivamente).

CONCLUSIONES: La afectación microscópica de la pared de la vena renal actúa como factor pronóstico dependiente para la supervivencia y para la progresión. La afectación macroscópica de la vena actúa como factor pronóstico independiente, aunque los pacientes que presentan ambas circunstancias lafectación macro y microscópica) son los que presentan peor evolución.

Palabras clave: Cáncer de riñón. Pronóstico. Vena renal.

Summary.- OBJECTIVES: International TNM Staging System for renal cell carcinoma (RCC) classifies as T3b when "fumor grossly extends into the renal vein or its segmental branches, or vena cava below the diaphragm". The finding of microscopic invasion of the vein wall is not taken into account for TNM staging. We analyse its prognostic significance in localized and locally advanced RCC. 
METHODS: From January 1989 to July 2002, 257 RCC were surgically excised. Excluding Von HippelLindau patients and stage IV (TNM 2002), 241 cases were studied in retrospect, with a median follow up of 50.96 months. Histopathological data from the renal vein were available in 216 specimens. There was renal vein wall invasion in 22. We compare the outcomes in this group vs. the group without microscopic involvement of the renal vein wall (n: 194).

RESULTS: Mean age for the group with renal vein invasion (RVI) was 65.02 years. Mean tumour size in the same group was $9 \mathrm{~cm}$, larger than in control group $(p<0,001)$. Thrombus was found in $72.7 \%$ vs. $6.2 \%$ in the control group. Clear cell carcinoma (77.3\%) was the predominant histological subtype. Nuclear grade 2 according to Fuhrman's Classification System accounts for $42.9 \%$ of the cases. Metastatic progression risk (HR: 4,86) and death risk (HR: 6,49) are significantly higher in RVI group. When renal vein thrombosis is found, progression and death risks are still higher (HR: 7.22 and 8.38, respectively).

CONCLUSIONS: Microscopic invasion of the renal vein wall is a dependent prognostic factor for disease progression and death for RCC. Macroscopic renal vein involvement is an independent prognostic factor. When both factors are found together, disease outcome is worse.

Keywords: Kidney cancer. Prognosis. Renal Vein.

\section{INTRODUCCIÓN}

Una característica del cáncer de células renales (CCR) es su tendencia a invadir el sistema venoso, el cual se encuentra afectado en un $10 \%$. El modo más frecuente, aunque no el único de manifestarse este hecho, es en forma de un trombo tumoral que puede extenderse dentro de la cava alcanzando incluso la aurícula derecha (1). La afectación vascular también puede ser microscópica, en este caso el porcentaje de afectación de la pared de la vena renal varía según las series, desde el $4,7 \%$ al $40,5 \%$ (2-7). Esta variabilidad en el porcentaje probablemente viene condicionada por la falta de unanimidad en la definición de invasión vascular microscópica (4). Algunos autores consideran que la infiltración microscópica (Figura 1) y la invasión de la vena renal por trombo intraluminal (Figura 2), representan dos mecanismos diferentes de invasión vascular. La primera implica una destrucción inmediata del endotelio y la segunda, tardía. Cuando la invasión es macroscópica el endotelio íntegro recubre la masa tumoral. Cuando la afectación es microscópica se puede facilitar la diseminación celular a través de la desestructuración endotelial, lo que condicionaría un peor pronóstico $(3,4)$. Sin embargo, su valor pronóstico en el CCR no está bien establecido. En la TNM del 2002 (8) se contempla la invasión microvascular del seno, considerándolo un T3a. Se recoge por primera vez la invasión de la pared de la cava, pero no establece con claridad qué consideran invasión de la misma.

En este trabajo estudiamos la asociación de la afectación microscópica de la vena renal con otras variables y su implicación pronóstica en la progresión y la supervivencia del cáncer renal localizado y localmente avanzado.

\section{MATERIAL Y MÉTODO}

De 257 tumores renales operados en el Hospital Clínico San Carlos (Madrid) entre Enero de 1989 y Julio de 2002, se excluyen los estadios IV (TNM 2002) y dos casos que presentan enfermedad de Von Hippel-Lindau, analizándose retrospectivamente 241 casos, con un seguimiento mediano de 50,96 meses. Tenemos datos de la histología de la vena en 216 casos, en un caso no hay datos y 24 corresponden a los intervenidos mediante cirugía conservadora. Consideramos que existe afectación microscópica de la vena renal, cuando se objetiva al microscopio infiltración tumoral de toda la pared vascular, incluyendo la capa endotelial. Comparamos el grupo con invasión de la pared de la vena renal (n: $22,10,2 \%$ ) frente el resto (n: $194,89,8 \%$ ).

Se estiman las funciones de supervivencia por el método de Kaplan-Meier, para las variables independientes para el estudio de muerte y progresión de la enfermedad. Se realiza un análisis estratificado para evaluar la existencia de interacciones. Se ajusta un modelo de regresión de riesgos proporcionales de Cox. El análisis estadístico se realiza mediante el programa SPSS, versión 12.0.

\section{RESULTADOS}

La afectación microscópica de la pared de la vena renal es más frecuente en el varón $(62,4 \%)$, aunque sin diferencias respecto al grupo sin afectación (p: 0,26). La edad media es de 65,02 años en el grupo con afectación y 63,12 años en el grupo sin afectación (p: 0,56).

El tamaño medio de los casos con afectación de la pared es significativamente mayor, $9 \mathrm{~cm}$ (SE: 0,95; rango: $2,5-19$ ) vs $5,7 \mathrm{~cm}$ (SE: 0,2; rango: 1 23) $(p<0,001)$. 
TABLA I. VARIABLES HISTOLÓGICAS ANALIZADAS EN LOS DOS GRUPOS DE ESTUDIO (CON Y SIN INVASIÓN MICROSCÓPICA DE LA PARED)

\begin{tabular}{|c|c|c|c|}
\hline Variables & Invasión de la pared (\%) & No invasión (\%) & $\mathbf{p}$ \\
\hline Tipo celular: & & & 0,45 \\
\hline - Células claras & 77,3 & 64,9 & \\
\hline - Papilar & 4,5 & 17,0 & \\
\hline - Cromófobo & 9,1 & 11,9 & \\
\hline - Tumor de Bellini & 4,5 & 4,1 & \\
\hline - Inclasificable & 4,5 & 2,1 & \\
\hline Presencia de más de un patrón de crecimiento & 23,8 & 44,7 & 0,05 \\
\hline Presencia de patrón sólido & 85,7 & 71,1 & 0,13 \\
\hline Presencia de patrón tubular & 19,0 & 43,2 & 0,02 \\
\hline Presencia de patrón papilar & 14,3 & 31,1 & 0,08 \\
\hline Presencia de patrón quístico & 14,3 & 10,5 & 0,61 \\
\hline Multifocalidad & 18,2 & 16 & 0,79 \\
\hline Histología sarcomatoide & 4,5 & 5,7 & 0,82 \\
\hline Necrosis & 50,0 & 41,2 & 0,43 \\
\hline Hemorragia intratumoral & 36,4 & 59,8 & 0,03 \\
\hline Calcificaciones & 0,0 & 11,3 & 0,02 \\
\hline Grado nuclear de Furhman & & & 0,62 \\
\hline-1 & 23,8 & 30,3 & \\
\hline-2 & 42,9 & 48,4 & \\
\hline-3 & 23,9 & 17,0 & \\
\hline-4 & 9,5 & 4,3 & \\
\hline Presencia de mitosis & 18,2 & 8,2 & 0,16 \\
\hline Afectación del sistema pielocalicial & 13,6 & 9,3 & 0,53 \\
\hline Afectación de la pared arterial & 9,1 & 1,0 & 0,04 \\
\hline Trombosis arterial & 13,6 & 1,0 & 0,005 \\
\hline Afectación de grasa perirrenal & 59,1 & 39,2 & 0,07 \\
\hline Afectación de la suprarrenal & 0,0 & 2,2 & 0,42 \\
\hline Presencia de trombo & & & $<0,001$ \\
\hline - No & 27,3 & 91,2 & \\
\hline - Vena Cava & 0,0 & 1,0 & \\
\hline - Vena Renal & 72,7 & 6,2 & \\
\hline - Intrarrenal & 6,2 & 1,5 & \\
\hline pT (TNM 2002) & & & $<0,001$ \\
\hline$-\mathrm{T} 1 \mathrm{a}$ & 9,1 & 29,4 & \\
\hline$-T 1 b$ & 4,5 & 22,2 & \\
\hline$-\mathrm{T} 2$ & 4,5 & 9,3 & \\
\hline- T3 a & 4,5 & 32,5 & \\
\hline- T3 b & 77,3 & 6,7 & \\
\hline $\mathrm{pN}$ & & & 0,05 \\
\hline$-\mathrm{NO}$ & 13,6 & 16,5 & \\
\hline$-N 1$ & 9,1 & 0,5 & \\
\hline$-N X$ & 77,3 & 83 & \\
\hline
\end{tabular}


TABLA II. PROBABILIDAD DE ESTAR LIBRE DE ENFERMEDAD A LOS 5 AÑOS EN LOS TUMORES SIN AFECTACIÓN MICROSCÓPICA DE LA PARED ANTE LA PRESENCIA O AUSENCIA DE TROMBO.

\begin{tabular}{|l|c|c|c|}
\hline & Pr\% & MEDIA & P \\
& EE & EE \\
IC 95\% & \\
\hline No afectación de la pared- & 46,18 & 54,77 & \\
Trombo en vena & 16,66 & 18,52 & $<0,001$ \\
& 3 & $(18,4-91,1)$ & \\
No afectación de la pared- & 85,67 & 139,37 & \\
No trombo en vena & 2,93 & 4,99 & \\
& 81 & $(129,6-149,1)$ & \\
\hline
\end{tabular}

Pr-\%: probabilidad en tanto por ciento. EE: Error estándar. N: número de casos. IC95\%: intervalo de confianza del 95\%

Analizamos diversas variables histológicas en ambos grupos (Tabla I). No encontramos diferencias significativas en cuanto al tipo celular. La presencia de más de un patrón de crecimiento es significativamente más frecuente en el grupo sin afectación, al igual que el patrón tubular. También en este grupo es más frecuente la presencia de hemorragia intratumoral y de calcificaciones. Por el contrario, la afectación de la pared arterial y de trombosis arterial, se objetiva con mayor frecuencia en el grupo con afectación microscópica de la pared, al igual, que la presencia de trombo en la vena renal. Las diferencias en el estadio, vienen condicionadas por el estadio $\mathrm{T} 3 \mathrm{~b}$, que es significativamente más frecuente en el grupo con afectación microscópica de la pared.

El 22,7\% con afectación microscópica de la pared, presentan recidiva local, porcentaje estadísticamente superior, que el grupo sin afectación $(4,1 \%)$ (p: 0,001). Lo mismo sucede con la progresión a distancia $(45 \%$ vs $15 \% ; \mathrm{p}: 0,003)$.

Tanto la presencia de trombo como la afectación microscópica de la pared actúan como factores

TABLA III. PROBABILIDAD DE ESTAR LIBRE DE ENFERMEDAD A LOS 5 AÑOS EN LOS TUMORES CON
AFECTACIÓN MICROSCÓPICA DE LA PARED ANTE LA PRESENCIA O AUSENCIA DE TROMBO.

\begin{tabular}{|l|c|c|c|}
\hline & Pr\% & MEDIA & P \\
& EE & EE & IC 95\% \\
& N & 36,71 & \\
\hline Afectación de la pared- & 17,14 & 8,25 & \multirow{2}{*}{0,20} \\
Trombo en vena & 14,91 & $(20,5-52,8)$ & \\
\hline Afectación de la pared- & 1 & 89,00 & \\
No trombo en vena & 80,00 & 18,25 & \\
& 17,89 & $(53,2-124,7)$ & \\
\hline
\end{tabular}

Pr-\%: probabilidad en tanto por ciento. EE: Error estándar. N: número de casos. IC95\%: intervalo de confianza del 95\% 
TABLA IV. ANÁLISIS MULTIVARIADO DE LA PROBABILIDAD DE ESTAR LIBRE DE ENFERMEDAD.

\begin{tabular}{|l|c|c|c|}
\hline & HR & IC 95\% & P \\
\hline Edad $\geq 65$ años & 3,34 & $0,6-13,4$ & 0,005 \\
\hline Leucocitos>10 $\times 109$ & 4,17 & $2,3-36,6$ & 0,007 \\
\hline Mitosis & 4,81 & $1,7-13,3$ & 0,003 \\
\hline Sarcomatoide & 5,04 & $1,7-46,0$ & 0,003 \\
\hline T 3b & 5,14 & $2,1-12,1$ & $<0,001$ \\
\hline
\end{tabular}

pronósticos dependientes para la progresión de la enfermedad. La presencia de trombo en la vena renal eleva el riesgo de progresión en 6,40 veces (IC95\%: $3,3-12,3 ; p<0,0011$, con una probabilidad de estar libre de enfermedad a los 5 años del $36,26 \%$. La afectación microscópica de la pared aumenta el riesgo en 3,89 veces (IC95\%: 1,84-8,24; p: 0,002) y la probabilidad de estar libre de enfermedad a los 5 años es del $40,15 \%$.

Sin embargo, cuando existe afectación venosa, la diferencia en el riesgo de progresión la marca la presencia o no de trombo, aunque los que presentan menos probabilidad de estar libre de progresión son los que tienen trombo y afectación microscópica de la pared (17,4\% a los 5 años) (Tablas II y III).
De hecho, al realizar el análisis multivariado para la progresión, la variable que se mantiene es el estadio T3b, perdiendo la significación la afectación microscópica (Tabla IV).

El tiempo de supervivencia es de $39,11 \mathrm{me}-$ ses (SE: 6,82; rango; 0,30- 109,40) en el grupo con afectación y de 65,50 meses (SE: 3,22; rango: 0,20-168,23) (p: 0,01) en el otro grupo. El 22,7\% del primer grupo mueren por causa tumoral, frente al $5,2 \%$ del grupo sin afectación (p: 0,009). La probabilidad de estar vivo a los 5 años en el grupo que tiene afectación de la pared es de 68,06\% frente al 96,03\% (HR: 6,49; IC95\%: 2,1-19,2) (p: 0,003) (Figura 3).

\section{TABLA V. PROBABILIDAD DE ESTAR VIVO EN LOS TUMORES SIN AFECTACIÓN DE LA PARED ANTE LA PRESENCIA O AUSENCIA DE TROMBO.}

\begin{tabular}{|l|c|c|c|}
\hline & Pr\% & MEDIA & P \\
& EE & EE & IC 95\% \\
\hline No afectación de la pared- & 78,75 & 105,80 & \\
Trombo en vena & 13,40 & 17,12 & \multirow{2}{*}{0,001} \\
& 3 & $(72,2-139,3)$ & \\
No afectación de la pared- & 96,95 & 160,05 & \\
No trombo en vena & 1,36 & 2,83 & \\
& 88 & $(154,4-165,6)$ & \\
\hline
\end{tabular}

Pr-\%: probabilidad en tanto por ciento. EE: Error estándar. N: número de casos. IC95\%: intervalo de confianza del $95 \%$ 


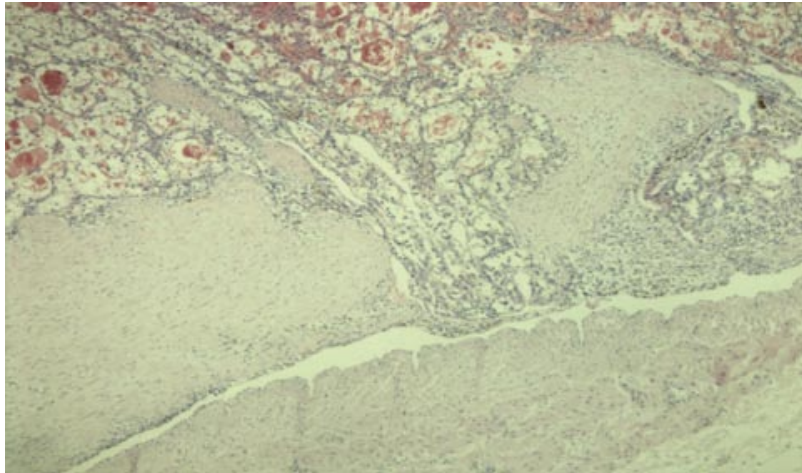

FIGURA 1. Infiltración tumoral de la capa muscular de la vena renal, alcanzando el endotelio (Hematoxilina-eosina $\times 5$ ).

La presencia de trombo en la vena renal eleva más el riesgo de muerte que la afectación de la pared (HR: 7,22; IC95\%: 2,5-20,5; p: 0,008). El trombo en la vena renal se asocia a mal pronóstico de forma significativa ( $p: 0,001)$, esté o no afectada la pared de la vena (Tabla V).

Cuando existe afectación de la pared, aunque no hay diferencias significativas en la supervivencia ante la presencia o no de trombo (p: 0,60$)$, ésta es peor en los casos en los que se asocia a trombo (probabilidad de estar vivo a los 5 años del 61,54\%) (Tabla VI).

Al igual que sucede con la progresión, esto concuerda con los resultados en el análisis multivariado, ya que es el estadio T3b (presencia de trombo),

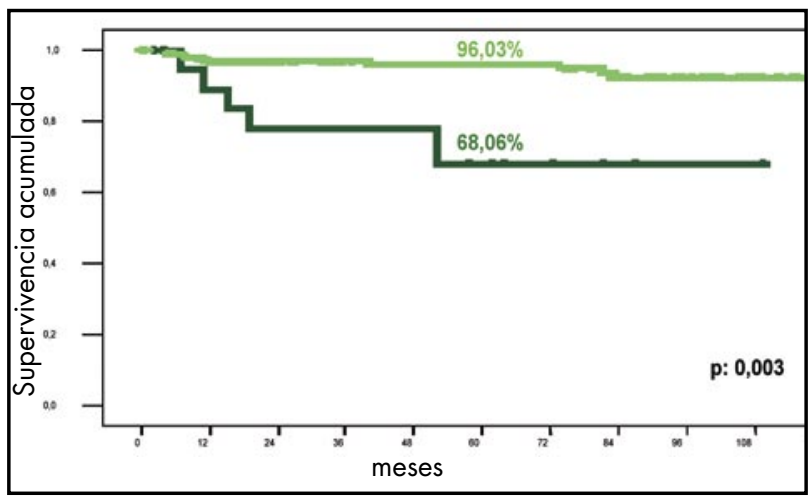

FIGURA 3. Probabilidad de estar vivo a los 5 años dependiendo de la afectación o no, microscópica de la pared de la vena.

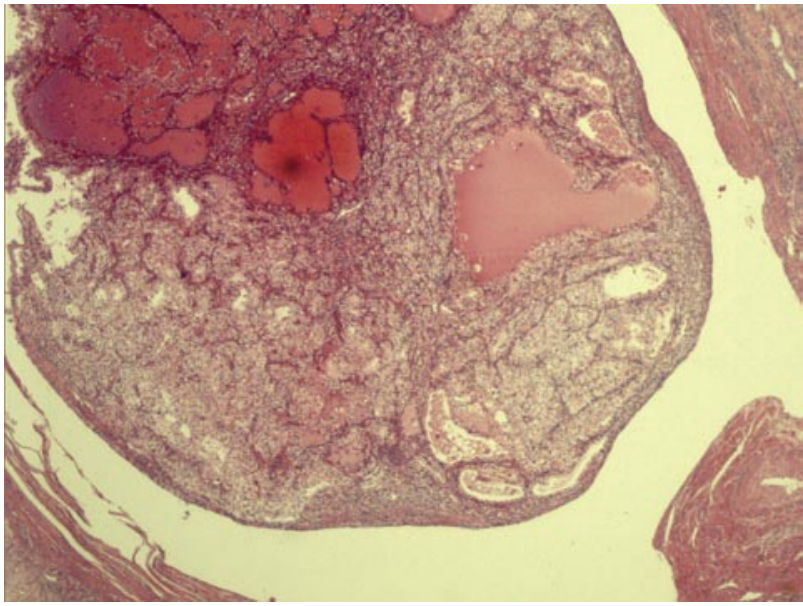

FIGURA 2. Trombo tumoral en la luz de la vena renal (Hematoxilina-eosina $\times 2,5$ ).

el que se mantiene como factor pronóstico independiente, elevando el riesgo de muerte en 9 veces (HR: 9,01 ; IC95\%: 1,6-48,5; p: 0,01), junto a la presencia de más de un patrón de crecimiento y el grado 4 de Fuhrman (HR: 9,04; IC95\%: 1,7-46,0; p: 0,008 y HR: 14,35; IC95\%: 2,9-70,7; p: 0,001, respectivamente).

\section{DISCUSIÓN}

El modo más frecuente de invasión del sistema venoso por el CCR es en forma de un trombo tumoral (1). En estos casos (invasión venosa macroscópica), el borde tumoral permanece rodeado por el endotelio (3). Pero, también se puede objetivar invasión microscópica. El porcentaje de afectación microscópica de la pared de la vena renal varía según las series, desde el $4,7 \%$ al $40,5 \%$ (2-7) y en otros trabajos, no aportan este dato $(9,10)$. En nuestro grupo la afectación se objetiva en el 10,2\%, porcentaje dentro de lo publicado.

Esta variabilidad en el porcentaje, probablemente viene condicionada por la falta de unanimidad en la definición de invasión vascular microscópica (4). Nosotros al igual que otros autores $(4,6)$ definimos la invasión microvascular como la infiltración tumoral de toda la pared de la vena, incluyendo la capa endotelial. Sevinç et al. (4) consideran que existe invasión si objetivan tumor en un vaso que conserva al menos una célula endotelial o cuando pueden reconocer la túnica media vascular alrededor de un grupo de células neoplásicas. Mrstik et al. (6) utilizan el anticuerpo antifactor (8) para identificar la invasión. Lang et al. (3) emplean el anticuerpo anti-CD34 y el anticuerpo antifactor (8); y consideran que éste 
último es más útil para el diagnóstico de la invasión vascular.

Parece que la afectación microscópica se asocia a otros factores de mal pronóstico. Se relaciona con el tamaño, el grado, $(3,4,7)$ el estadio $(2,3$, 7) y con la histología sarcomatoide $(3,4)$.

A mayor tamaño, mayor es el grado de afectación, superando el $50 \%$ en los mayores de $10 \mathrm{~cm}$, siendo las diferencias estadísticamente significativas $(4,7)$. En nuestro grupo el tamaño medio de los casos con afectación de la pared es significativamente mayor $(9 \mathrm{~cm}$ vs $5,7 \mathrm{~cm} ; \mathrm{p}<0,001)$. En el trabajo de Lang et al. (3) la invasión microvascular disminuye de forma significativa la supervivencia en los tumores con un tamaño mayor de $10 \mathrm{~cm}$.

Tanto en el trabajo de Sevinç et al. (4) como en el lang et al. (3) la invasión de la pared se asocia a los tumores con histología sarcomatoide de forma estadísticamente significativa, determinando peor pronóstico. En nuestro grupo no encontramos diferencias $(4,5 \%$ vs $5,7 \%$; p: 0,82$)$.

Parece que el porcentaje de afectación también aumenta con el grado nuclear $(4,7)$. La diferencias son significativas (7), aunque en algún estudio la diferencia es entre el grado III, respecto al I y II (4). En otro estudio disminuye la supervivencia en el grado IV de Fuhrman (p: 0,035) (3). En nuestro trabajo, aunque no hay diferencias en el grado ( $p: 0,62)$, el grupo con afectación microscópica presentan un porcentaje de grados 4 superior $(9,5 \%$ vs $4,3 \%)$.

Para Lang et al. (3) la invasión microvascular también disminuye la supervivencia en los tumores con invasión capsular (p: 0,012) e invasión de la vena renal (se supone que se refieren a la afectación macroscópica) (p: 0,05). En nuestro trabajo la afectación microscópica se da con más frecuencia en los casos con trombo tumoral, pero no objetivamos esta asociación con la afectación capsular.

En cuanto al estadio, Sevinç et al. (4) encuentran invasión en el $11 \%$ de los $\mathrm{T} 1$ y en el $29 \%$ de los T2 (TNM 1997), sin diferencias significativas. En nuestro trabajo el estadio más afectado es el T3b $(77,3 \%)$, en el resto de los estadios el porcentaje es muy inferior, aunque llama la atención que el porcentaje de los Tla sea superior al resto $19,1 \%$ frente al $4,5 \%$ ). En el estudio de Lang et al. (3) es en los T3a-b donde se marca la diferencia en la supervivencia (p: $0,012)$, siguiendo la clasificación de 1992. Hemanek y Schrott (5), objetivan que en los tumores T2 (TNM 1987) con afectación microscópica de la vena presentan una menor supervivencia a los 5 años, actuando esta afectación como un factor pronóstico. Presentan una supervivencia del $94 \%$ sin afectación y del $77 \%$ con afectación. Esto no lo objetivan en los T3. En el análisis excluyen a los T1 por ser un número muy reducido, tanto con como sin afectación 4 y 7 casos, respectivamente).

La afectación de la pared podría ser un factor pronóstico más importante que la extensión del trombo hacia la cava supradiafragmática. Según Hatcher et al. (11) los pacientes con trombo en cava (no diferencian entre supra e infradiafragmática) sin afectación de la pared, alcanzan una supervivencia del $69 \%$ a los 5 años. Con infiltración de la cava desciende al $26 \%$ (p: 0,04). Sin embargo, con la exéresis completa del segmento de cava infiltrado aumenta la supervivencia al 57\%. Por el contrario, Ljungberg et al. (12) no encuentran diferencias significativas en cuanto a la supervivencia entre los que presentan invasión de la pared y los que la tienen libre.

Su valor pronóstico en el CCR no está bien establecido. En la TNM del 2002 (8) se reconoce la invasión microvascular del seno renal, la localización más frecuente, considerándolo un T3a. (13). Se recoge por primera vez la invasión de la pared de la cava, pero no se establece con claridad qué consideran invasión de la misma. Tampoco, si todo paciente con afectación de la pared de la cava se considera T3c, o únicamente los que presentan afectación supradiafragmática. Teniendo en cuenta que puede existir afectación de la pared de la vena sin presencia de trombo, y esto no se puede detectar con certeza macroscópicamente en la mayoría de los casos, nos hace presumir que la afectación ha de ser microscópica.

Algunos autores consideran que la invasión microscópica tiene una influencia pronóstica significativa $(2$, $7,14)$, aunque no siempre independiente $(3-6,15)$.

Para Van Poppel et al. (7) la presencia de invasión vascular microscópica es el factor pronóstico más importante, actuando de forma independiente en el CCR no diseminado; pero no comparan la significación de la invasión microvascular respecto a la afectación por trombo de la vena renal. Tampoco incluyen a los estadios T1 y T4 (TNM 1987), al contar con muy pocos casos.

Griffiths et al. (16) en una serie de 176 pacientes con CCR localizado o localmente avanzado, dividen la invasión vascular en 3 categorías: invasión de la cava inferior, invasión de los vasos hiliares principales, invasión vascular objetivada microscópicamente en vasos que no sean los hiliares ni la cava. No aclaran la presencia o ausencia de 
trombo. Presentan invasión microvascular 24 casos, sin otros datos de afectación vascular. De los 31 que presentan invasión macroscópica de la vena renal, 21 de ellos también presentan invasión microvascular. Al realizar el modelo de regresión de Cox, los factores predictivos independientes son el grado, la edad y el nivel de invasión vascular. En cuanto a la invasión vascular, cualquier tipo de las afectaciones presentan un riesgo mayor que la ausencia de afectación, aunque la invasión microvascular no alcanza la significación estadística (HR: 2, 15; p: 0,057), el riesgo mayor es por la afectación de la cava inferior. El tamaño y el tipo histológico no resultan ser factores independientes y los autores dan como explicación la correlación vascular, ya que, los tumores con invasión vascular presentan un tamaño significativamente mayor y en cuanto al tipo histológico los carcinomas papilares presentan en una pequeña proporción invasión vascular.

Algunos autores objetivan que la invasión microscópica disminuye la supervivencia $(6,7,14)$. Lang et al. (3) únicamente encuentran que la afectación microscópica de la vena influye en la supervivencia durante el primer año $183,3 \%$ frente a $95,1 \%$ en el grupo sin afectación; p: 0,01). También objetivan que cuando se asocia a otros factores de mal pronóstico, la afectación microscópica acorta más la supervivencia y apuntan que, por tanto, se debería tener en cuenta a la hora de establecer un tratamiento adyuvante en el CCR localizado.

En el trabajo de Sevinç et al. (4) la tasa de supervivencia de la serie es del $81 \%$, con un segui- miento medio de 48 meses. Los que presentan afectación de la vena $70 \%$, y $85 \%$ para el otro grupo (p: $0,031)$. Con estos resultados sugieren que la invasión microscópica vascular se relaciona con el pronóstico, sin embargo, en el análisis multivariante no actúa como un factor pronóstico independiente. En nuestra serie la afectación de la pared implica mal pronóstico (HR: 6,49; p: 0,003), pero pierde la significación al realizar el análisis multivariado. Sin embargo, el trombo en la vena renal se asocia a mal pronóstico de forma significativa ( $p: 0,001$ ), esté o no afectada la pared de la vena (Tabla V). Pero cuando se dan las dos circunstancias (afectación de la pared y trombo), aunque las diferencias no son significativas, la probabilidad de estar vivo es menor (a los 5 años del 61,54\%) (Tabla VI).

También se relaciona con el desarrollo de metástasis $(3,6,7,14)$. Mrstik et al. (6) observan afectación microscópica en el $80 \%$ de los pacientes con metástasis. Sevinç et al. (4) objetivan que de los 34 pacientes sin afectación, 6 progresan $(17 \%)$ en un tiempo medio de 41 meses (rango 2-45), con afectación de la vena progresan 2 de los $7(29 \%)$ en una media de 3 meses (rango 1-5) (p: 0,01). Señalan que el desarrollo de metástasis es significativamente superior en este grupo ( $p: 0,01)$, aunque al igual que en nuestro estudio, la significación se pierde en el análisis multivariado.

En nuestro grupo, la probabilidad de estar libre de enfermedad a los 5 años cuando existe afectación microscópica es $40,15 \%$ frente al $83,68 \%$ del grupo sin afectación. Los que presentan afectación

TABLA VI. PROBABILIDAD DE ESTAR VIVO EN LOS TUMORES CON AFECTACIÓN DE LA PARED ANTE LA PRESENCIA O AUSENCIA DE TROMBO.

\begin{tabular}{|l|c|c|c|}
\hline & Pr\% & MEDIA & \\
& EE & EE \\
IC 95\% & P \\
\hline Afectación de la pared- & 61,54 & 64,25 & \\
Trombo en vena & 16,64 & 9,43 & \multirow{2}{*}{0,60} \\
& 4 & $(45,7-82,7)$ & \\
Afectación de la pared- & 80,00 & 91,32 & \\
No trombo en vena & 17,89 & 16,17 & \\
& 2 & $(59,6-123,0)$ & \\
\hline
\end{tabular}

Pr-\%: probabilidad en tanto por ciento. EE: Error estándar. N: número de casos. IC95\%: intervalo de confianza del $95 \%$ 
de la pared multiplican el riesgo casi 4 veces $(H R$ : 3,89; p: 0,002), pero únicamente actúa como factor pronóstico dependiente. Al realizar el análisis estratificado, la diferencia la marca la presencia o no de trombo, pero los que tiene menos probabilidad de estar libre de enfermedad a los 5 años, son los que presentan trombo y afectación de la pared $(17,4 \%)$ (Tablas II y III).

\section{CONCLUSIONES}

La afectación microscópica de la pared de la vena renal actúa como factor pronóstico dependiente en el CCR, tanto para la supervivencia como para la progresión. La afectación macroscópica de la vena sí que actúa como factor pronóstico independiente, aunque los pacientes que presentan ambas circunstancias (afectación macro y microscópica) son en los que se registran peores resultados.

Es preciso establecer una definición homogénea de afectación microvascular y recogerse de forma sistemática en el informe anatomopatológico. Con ello, se podrá establecer comparación entre diferentes grupos y asignar su implicación pronóstica, y por tanto, la necesidad o no de ser reflejado en la TNM.

\section{BIBLIOGRAFÍA y LECTURAS RECOMENDADAS (*lectura de interés $y^{* *}$ lectura fundamental)}

1. Novick AC and Campbell SC. Renal tumors en: Campbell's Urology, ed 8. Editado por Walsh PC. Philadelphia: WB Saunders, 2002; 2672-2731.

*2. Sorbellini M, Kattan MW, Snyder MA, Reuter $\mathrm{V}$, Motzer R, Goetzi M, et al. A postoperatitve prognostic nomogram predicting recurrence for patients with conventional clear cell renal cell carcinoma. J Urol, 2005; 173: 48-51.

**3. Lang H, Lindner V, Saussine C, Havel D, Faure F, Jacqmin D. Microscopic venous invasion: a pronostic factor in renal cell carcinoma. Eur Urol, 2000; 38: 600-605.

**4. Sevinç M, Kirkali Z, Yörükoglu K, Mungan Ugur, Sade Murat. Prognostic significance of microvascular invasion in localized renal cell carcinoma. Eur Urol, 2000; 38 (6): 728-33.
5. Hermanek P and Schrott KM. Evaluation of the new tumor, nodes and metastases classification of renal cell carcinoma. J Urol, 1990; 144: 238-241.

**6. Mrstik CH, Salomon J, Weber R, Stögermayer F. Microscopic venous infiltration as predictor of relapse in renal cell carcinoma. J Urol, 1992; 148 (2 Pt 1): 271-274.

**7. Van Poppel H, Vandendriessche H, Boel K, Mertens V, Goethuys H, Haustermans K, Van Damme B, Baert L. Microscopic vascular invasion is the most relevant prognosticator after radical nephrectomy for clinically nonmetastatic renal cell carcinoma. J Urol, 1997; 158: 45-49.

8. Greene FL, Page DL, Fleming ID, Fritz A, Balch CM, Haller DG, et al: AJCC Cancer Staging Manual, 6th ed. New York: Springer-Verlag, 2002; 21.

9. Ortiz M, Prados FJ, Rosales JL, Honrubia B, Martínez A, Cózar JM, Espejo E, Tallada M. Valoración de factores pronósticos de la supervivencia en una serie de 202 pacientes intervenidos por carcinoma de células renales. Actas Urol Esp, 2005; 29 (2): 179-189.

10. Sosa RE, Muecke EC, Darracot Vaughan Jr E, Mc Carron Jr JP. Renal cell carcinoma extending into the inferior vena cava: The prognostic significance of the level of the vena caval involvement. J Urol, 1984; 132: 1097-1100.

11. Hatcher PA, Anderson EE, Paulson PF, Carson CC, Robertson JE. Surgical management and prognosis of renal cell carcinoma invading the vena cava. J Urol, 1991; 145: 20-24.

*12. Ljungberg B, Stenling R, Osterdahl B, Farrelly E, Abers T, Roos G. Vein invasion of renal cell carcinoma: impact on metastatic behavior and survival. J Urol, 1995; 154: 1681-4.

13. Fleming $S$ and Griffiths DFR. Nephrectomy for renal tumour; dissection guide and dataset. J Clin Pathol, 2005; 58: 7-14.

*14. Javidan J, Stricker HJ, Tamboli P, Amin MB, Peabody JO, Deshpande A, et al. Prognostic significance of the 1997 TNM classification of renal cell carcinoma. J Urol, 1999; 162: 1277-1281.

15. Dimopoulos MA, Logothetis CJ, Markowitz A, Sella A, Amato R, Ro J. Collecting Duch carcinoma of the kidney. BJU 1993; 71: 388-391.

16. Griffiths DFR, Verghese A, Golash A, Kynaston HG, Matthews PN, Hart AJL, Courts JB. Contribution of grade, vascular invasion and age to outcome in clinically localized renal cell carcinoma. BJU International 2002; 90, 26-31. 